\title{
A Fourth Order Improved Numerical Scheme for the Generalized Burgers - Huxley Equation
}

\author{
Athanassios G. Bratsos \\ Department of Mathematics, Technological Educational Institution of Athens, Athens, Greece \\ E-mail:bratsos@teiath.gr \\ Received March 30, 2011; revised May 5, 2011; accepted May 22, 2011
}

\begin{abstract}
A fourth order finite-difference scheme in a two-time level recurrence relation is proposed for the numerical solution of the generalized Burgers-Huxley equation. The resulting nonlinear system, which is analyzed for stability, is solved using an improved predictor-corrector method. The efficiency of the proposed method is tested to the kink wave using both appropriate boundary values and conditions. The results arising from the experiments are compared with the relevant ones known in the available bibliography.
\end{abstract}

Keywords: Burgers-Huxley; Finite-Difference Method; Modified Predictor-Corrector

\section{Introduction}

A. Hodgkin and A. Huxley [1] proposed a model, known henceforth as the Huxley equation, in order to explain the ionic mechanisms underlying the initiation and propagation of action potentials in the squid giant axon. The most general form of the Huxley equation, known as the generalized Burgers-Huxley equation $(\mathrm{BgH})[2,3]$, has the form [4]

$$
u_{t}+\alpha u^{\delta} u_{x}-u_{x x}=\beta u\left(1-u^{\delta}\right)\left(u^{\delta}-\gamma\right) ; 0 \leq x \leq 1, t>0,
$$

where $u=u(x, t)$ is a sufficiently often differentiable function, $\alpha$ a real parameter, $\beta \geq 0, \gamma \in(0,1)$ and $\delta>0$. Equation (1.1), which models the interaction between reaction mechanisms, convection effects and diffusion transport, is the modified Burgers equation for $\beta=0$ (see [5] and the references therein), is also the Huxley equation [1] for $\alpha=0, \delta=1$ and is the Fitzhugh-Nagoma equation [6] for $\alpha=0$.

Many researchers have used various methods to solve the $\mathrm{BgH}$ equation. A theoretical study of the $\mathrm{BgH}$ equation was found in Wang et al. [4], while analytical solutions using various techniques in [7-11], etc., have been proposed. As far as the numerical methods are concerned among others the Adomian decomposition method was used by Ismail et al. [12] for the $\mathrm{BgH}$ and the Burgers-Fisher equation, and by Hashim et al. [13] for the $\mathrm{BgH}$ equation. Javidi [14] used the pseudospectral method, while Javidi [15], Javidi and Golbabai [16] the spectral collocation method. Batiha et al. [17] used the variational iteration method and Khattak [18] the collocation method with radial basis functions. Babolian and Saeidian [19] used the homotopy analysis method, etc.

The initial condition associated with Equation (1.1) will be

$$
u(x, 0)=f(x) ; 0 \leq x \leq 1 .
$$

\section{Theoretical Solution}

It is known [4] that Equation (1.1) has the following kink wave solution

$$
u(x, t)=\left\{\frac{\gamma}{2}+\frac{\gamma}{2} \tanh [k(x-c t)]\right\}^{1 / \delta}
$$

in which $k=\frac{\gamma \delta\left[-\alpha+\sqrt{\alpha^{2}+4 \beta(\delta+1)}\right]}{4(\delta+1)}$

and

$$
c=\frac{\alpha \gamma}{\delta+1}-\frac{(1+\delta-\gamma)\left[-\alpha+\sqrt{\alpha^{2}+4 \beta(\delta+1)}\right]}{2(\delta+1)}
$$

are the wave number and the velocity respectively.

\section{The numerical Method}

\subsection{Development of the Method}

\subsubsection{Grid and Solution Vector}

To obtain numerical solutions the region $R=\{(x, t) \in$ $[0<x<1] \times] 0, T]\}$ with its boundary $\partial R$ consisting of 
the lines $x=0, x=1$ and $t=0$ is covered with a rectangular mesh of points, $G$, with co-ordinates $(x, t)$ $=\left(x_{m}, t_{n}\right)=(m h, n \ell)$ with $m=0,1, \cdots, N+1$. The theoretical solution of Equation (1.1) at the typical mesh point $\left(x_{m}, t_{n}\right)$ will be denoted by $u_{m}^{n}$ and the relevant of an approximating difference scheme by $U_{m}^{n}$.

Let the solution vector at time level $t=t_{n}$ be

$$
U(t)=U^{n}=\left[U_{0}^{n}, U_{1}^{n}, \cdots, U_{N+1}^{n}\right]^{\mathrm{T}} .
$$

\subsubsection{Boundaries}

The following were used:

1) The space derivatives at the left boundary $x=0$ were replaced with second order finite-difference replacements of the form ([20] p. 17)

$$
\begin{aligned}
& \left.\left.u_{x}\right|_{x=0}=\frac{1}{2 h}\left(-3 U_{0}^{n}+4 U_{1}^{n}-U_{2}^{n}\right)+\mathcal{O}\left(h^{2}\right) \text { as } h \rightarrow 0, \text {, } 2.2\right) \\
& \left.u_{x x}\right|_{x=0}=\frac{1}{h^{2}}\left(2 U_{0}^{n}-5 U_{1}^{n}+4 U_{2}^{n}-U_{3}^{n}\right)+\mathcal{O}\left(h^{2}\right) \text { as } h \rightarrow 0
\end{aligned}
$$

and with analogous replacements to the right boundary $x=1$.

2) The boundary conditions

$$
\begin{aligned}
& u(0, t)=g_{0}(t) \text { and } u(1, t)=g_{1}(t) \\
& \left.u_{x}\right|_{x=0,1}=0 ; t>0,
\end{aligned}
$$

were used, while at the other interior points of the grid $G$ the well-known approximants based on the central-difference formulas.

\subsection{The Proposed Method}

Applying Equation (1.1) at each point of the grid $G$ at time level $t=t_{n}=n \ell ; n=1,2, \cdots$ leads to a first-order initial-value problem, which is written in a matrix-vector form as

$$
\begin{aligned}
& D \boldsymbol{U}(t)=-\alpha \Delta A \boldsymbol{U}(t)+B \boldsymbol{U}(t)+\beta \tilde{\Delta} \boldsymbol{U}(t) ; t>0 \\
& \boldsymbol{U}^{0}=\boldsymbol{U}(0)=\left[f\left(x_{0}\right), f\left(x_{1}\right), \cdots, f\left(x_{N+1}\right)\right]^{\mathrm{T}}=\boldsymbol{f}
\end{aligned}
$$

in which $D=\operatorname{diag}\{\mathrm{d} / \mathrm{d} t\}$,

$$
\begin{aligned}
\Delta & =\Delta^{n}=\Delta(t)=\operatorname{diag}\left\{\Delta_{m}^{n}\right\}=\operatorname{diag}\left\{\left(U_{m}^{n}\right)^{\delta}\right\}, \\
\tilde{\Delta} & =\tilde{\Delta}^{n}=\tilde{\Delta}(t)=\operatorname{diag}\left\{\tilde{\Delta}_{m}^{n}\right\} \\
& =\operatorname{diag}\left\{\left[1-\left(U_{m}^{n}\right]^{\delta}\right]\left[\left(U_{m}^{n}\right)^{\delta}-\gamma\right]\right\} ;
\end{aligned}
$$

for $m=0,1, \cdots, N+1$ are diagonal matrices,

$$
\begin{gathered}
A=\frac{1}{2 h}\left[\begin{array}{rrrrrr}
-3 & 4 & -1 & & & \\
-1 & 0 & 1 & & & \\
& \cdot & \cdot & \cdot & & \\
& & & -1 & 0 & 1 \\
& & & -1 & 4 & -3 \\
& & & & &
\end{array}\right], \\
B=\frac{1}{h^{2}}\left[\begin{array}{rrrrrrr}
2 & -5 & 4 & -1 & & & \\
1 & -2 & 1 & & & & \\
& \cdot & \cdot & \cdot & & & \\
& & & & 1 & -2 & 1 \\
& & & -1 & 4 & -5 & 2 \\
& & & & &
\end{array}\right]
\end{gathered}
$$

or

$$
\begin{aligned}
& A=\frac{1}{2 h}\left[\begin{array}{rrrrrr}
-2 & 2 & & & & \\
-1 & 0 & 1 & & & \\
& \cdot & \cdot & \cdot & & \\
& & & -1 & 0 & 1 \\
& & & & 2 & -2 \\
& & & & &
\end{array}\right], \\
& B=\frac{1}{h^{2}}\left[\begin{array}{rrrrrr}
-2 & 2 & & & & \\
1 & -2 & 1 & & & \\
& \cdot & \cdot & \cdot & & \\
& & & 1 & -2 & 1 \\
& & & & 2 & -2
\end{array}\right]
\end{aligned}
$$

tridiagonal matrices arising from the use of the boundary values (2.2) - (2.3) or the boundary condition (2.5) respectively and $\boldsymbol{f}$ the vector of the initial condition, all of order $N+2$.

Relation (2.6) gives

$$
D=-\alpha \Delta A+B+\beta \tilde{\Delta}
$$

hence $D^{2}$ can easily be obtained.

Using the recurrence relation

$$
\mathbf{U}(t+\ell)=\exp (\ell D) \mathbf{U}(t) ; t=0, \ell, \cdots,
$$

where $D \boldsymbol{U}(t)$ is given by (2.6) and replacing the matrix-exponential term with the fourth order rational approximant ([21] p. 134) gives

$$
\begin{aligned}
& \left(I-\frac{1}{2} \ell D+\frac{1}{12} \ell^{2} D^{2}\right) \mathbf{U}(t+\ell) \\
= & \left(I+\frac{1}{2} \ell D+\frac{1}{12} \ell^{2} D^{2}\right) \mathbf{U}(t) .
\end{aligned}
$$

Equation (2.13) using the notations (2.7) - (2.8) and Equation (2.11) leads to the following nonlinear system 


$$
\begin{aligned}
& \mathbf{U}(t+\ell)-\frac{1}{2} \ell\left(-\alpha \Delta^{n+1} A+B+\beta \tilde{\Delta}^{n+1}\right) \mathbf{U}(t+\ell) \\
& +\frac{1}{12} \ell^{2}\left[\left(\alpha \Delta^{n+1} A\right)^{2}+B^{2}+\left(\beta \tilde{\Delta}^{n+1}\right)^{2}-\alpha \Delta^{n+1} A B\right. \\
& -\alpha \beta \Delta^{n+1} A \tilde{\Delta}^{n+1}-\alpha B \Delta^{n+1} A+\beta B \tilde{\Delta}^{n+1} \\
& \left.-\alpha \beta \tilde{\Delta}^{n+1} \Delta^{n+1} A+\beta \tilde{\Delta}^{n+1} B\right] \mathbf{U}(t) \\
& =\mathbf{U}(t)+\frac{1}{2} \ell\left(-\alpha \Delta^{n} A+B+\beta \tilde{\Delta}^{n}\right) \mathbf{U}(t) \\
& +\frac{1}{12} \ell^{2}\left[\left(\alpha \Delta^{n} A\right)^{2}+B^{2}+\left(\beta \tilde{\Delta}^{n}\right)^{2}-\alpha \Delta^{n} A B\right. \\
& -\alpha \beta \Delta^{n} A \tilde{\Delta}^{n}-\alpha B \Delta^{n} A+\beta B \tilde{\Delta}^{n} \\
& \left.-\alpha \beta \tilde{\Delta}^{n} \Delta^{n} A+\beta \tilde{\Delta}^{n} B\right] \mathbf{U}(t) .
\end{aligned}
$$

Let

$r_{1}=\ell \alpha / 4 h, r_{2}=\ell / 2 h^{2}, r_{3}=\ell \beta / 2$,

$r_{4}=\ell^{2} \alpha^{2} / 48 h^{2}, r_{5}=\ell^{2} / 12 h^{4}, r_{6}=\ell^{2} \beta^{2} / 12$,

$r_{7}=\ell^{2} \alpha / 24 h^{3}, r_{8}=\ell^{2} \alpha \beta / 24 h$ and $r_{9}=\ell^{2} \beta / 12 h^{2}$.

Equation (2.14), when applied to the general mesh point of the grid $G$, gives

$$
\begin{aligned}
& U_{m}^{n+1}+r_{1} \Delta_{m}^{n+1}\left(-U_{m-1}^{n+1}+U_{m+1}^{n+1}\right)-r_{2}\left(U_{m-1}^{n+1}-2 U_{m}^{n+1}+U_{m+1}^{n+1}\right) \\
& -r_{3} \tilde{\Delta}_{m}^{n+1} U_{m}^{n+1}+r_{4} \Delta_{m}^{n+1}\left[\Delta_{m-1}^{n+1} U_{m-2}^{n+1}+\Delta_{m+1}^{n+1} U_{m+2}^{n+1}\right. \\
& \left.-\left(\Delta_{m-1}^{n+1}+\Delta_{m+1}^{n+1}\right) U_{m}^{n+1}\right]+r_{5}\left(U_{m-2}^{n+1}-4 U_{m-1}^{n+1}+6 U_{m}^{n+1}\right. \\
& \left.-4 U_{m+1}^{n+1}+U_{m+2}^{n+1}\right)+r_{6}\left(\tilde{\Delta}_{m}^{n+1}\right)^{2} U_{m}^{n+1}-r_{7} \Delta_{m}^{n+1}\left(-U_{m-2}^{n+1}\right. \\
& \left.+2 U_{m-1}^{n+1}-2 U_{m+1}^{n+1}+U_{m+2}^{n+1}\right)-r_{8} \Delta_{m}^{n+1}\left(-\tilde{\Delta}_{m-1}^{n+1} U_{m-1}^{n+1}\right. \\
& \left.+\tilde{\Delta}_{m+1}^{n+1} U_{m+1}^{n+1}\right)-r_{7}\left[-\Delta_{m-1}^{n+1} U_{m-2}^{n+1}+2 \Delta_{m}^{n+1}\left(U_{m-1}^{n+1}-U_{m+1}^{n+1}\right)\right. \\
& \left.+\Delta_{m+1}^{n+1} U_{m+2}^{n+1}+\left(\Delta_{m-1}^{n+1}-\Delta_{m+1}^{n+1}\right) U_{m}^{n+1}\right]+r_{9}\left(\tilde{\Delta}_{m-1}^{n+1} U_{m-1}^{n+1}\right. \\
& \left.-2 \tilde{\Delta}_{m}^{n+1} U_{m}^{n+1}+\tilde{\Delta}_{m+1}^{n+1} U_{m+1}^{n+1}\right)-r_{8} \Delta_{m}^{n+1} \tilde{\Delta}_{m}^{n+1}\left(-U_{m-1}^{n+1}+U_{m+1}^{n+1}\right) \\
& +r_{9} \tilde{\Delta}_{m}^{n+1}\left(U_{m-1}^{n+1}-2 U_{m}^{n+1}+U_{m+1}^{n+1}\right) \\
= & U_{m}^{n}-r_{1} \Delta_{m}^{n}\left(-U_{m-1}^{n}+U_{m+1}^{n}\right)+r_{2}\left(U_{m-1}^{n}-2 U_{m}^{n}+U_{m+1}^{n}\right) \\
+ & r_{3} \tilde{\Delta}_{m}^{n} U_{m}^{n}+r_{4} \Delta_{m}^{n}\left[\Delta_{m-1}^{n} U_{m-2}^{n}+\Delta_{m+1}^{n} U_{m+2}^{n}-\left(\Delta_{m-1}^{n}+\Delta_{m+1}^{n}\right)\right. \\
+ & \left.U_{m}^{n}\right]+r_{5}\left(U_{m-2}^{n}-4 U_{m-1}^{n}+6 U_{m}^{n}-4 U_{m+1}^{n}+U_{m+2}^{n}\right)+r_{6}\left(\tilde{\Delta}_{m}^{n}\right)^{2} U_{m}^{n} \\
- & r_{7} \Delta_{m}^{n}\left(-U_{m-2}^{n}+2 U_{m-1}^{n}-2 U_{m+1}^{n}+U_{m+2}^{n}\right) \\
- & r_{8} \Delta_{m}^{n}\left(-\tilde{\Delta}_{m-1}^{n} U_{m-1}^{n}+\tilde{\Delta}_{m+1}^{n} U_{m+1}^{n}\right)-r_{7}\left[-\Delta_{m-1}^{n} U_{m-2}^{n}\right. \\
+ & \left.2 \Delta_{m}^{n}\left(U_{m-1}^{n}-U_{m+1}^{n}\right)+\Delta_{m+1}^{n} U_{m+2}^{n}+\left(\Delta_{m-1}^{n}-\Delta_{m+1}^{n}\right) U_{m}^{n}\right] \\
+ & r_{9}\left(\tilde{\Delta}_{m-1}^{n} U_{m-1}^{n}-2 \tilde{\Delta}_{m}^{n} U_{m}^{n}+\tilde{\Delta}_{m+1}^{n} U_{m+1}^{n}\right)-r_{8} \Delta_{m}^{n} \tilde{\Delta}_{m}^{n}\left(-U_{m-1}^{n}+U_{m+1}^{n}\right) \\
+ & r_{9} \tilde{\Delta}_{m}^{n}\left(U_{m-1}^{n}-2 U_{m}^{n}+U_{m+1}^{n}\right) ; \\
&
\end{aligned}
$$$$
m=0,1, \ldots, N+1 \text {. }
$$

\section{Stability Analysis}

Following the Fourier method of analysing stability ([21] p. 142) if $\xi=e^{\kappa \ell}$ is the amplification factor and $\tilde{U}_{m}^{n}$ the numerical value of $U_{m}^{n}$ actually obtained, an error of the form $U_{m}^{n}-\tilde{U}_{m}^{n}=\xi^{n} e^{i m \mu h} ; i=\sqrt{-1}$ with $\kappa$ a complex number and $\mu$ real is considered. Then Equation (2.15) leads to the following stability equation

$$
\begin{aligned}
& {\left[1-r_{1} \tilde{\Delta}_{0}+4 r_{3} \sin ^{2} \omega+2 i r_{2} \Delta_{0} \sin 2 \omega\right] \xi} \\
& =1+2 r_{1} \tilde{\Delta}_{0}+r_{6} \tilde{\Delta}_{0}^{2}-8\left(r_{3}+r_{9} \tilde{\Delta}_{0}\right) \sin ^{2} \omega+16 r_{5} \sin ^{4} \omega \\
& -4 r_{4} \Delta_{0}^{2} \sin ^{2} 2 \omega+4 i \Delta_{0}\left[\left(-r_{2}+2 r_{7}-r_{8} \tilde{\Delta}_{0}\right) \sin 2 \omega\right. \\
& \left.-r_{7} \sin 4 \omega\right]
\end{aligned}
$$

where $U_{0}$ a typical value of $U_{m}^{n+1}, U_{m}^{n} ; m=0,1, \cdots$, $N+1$ used for the linearization of the nonlinear terms, $\Delta_{0}=U_{0}^{\delta}, \quad \tilde{\Delta}_{0}=\left(1-\Delta_{0}\right)\left(\Delta_{0}-\gamma\right)$ and $\omega=\beta h / 2$ with $\omega \in[0, \pi / 2]$. Equation (2.16) is of the form

$$
\breve{A} \xi=\breve{B} ; \breve{A}, \breve{B} \in \mathcal{C}
$$

with $\mathcal{C}$ the set of the complex numbers, so the von Neumann necessary criterion for stability $|\xi| \leq 1$ will always be satisfied when

$$
|\breve{B}| \leq|\breve{A}| \text {. }
$$

Inequality (2.18) for $\omega=0$ leads to

$$
\left|1+2 r_{1} \tilde{\Delta}_{0}+r_{6} \tilde{\Delta}_{0}^{2}\right| \leq\left|1-r_{1} \tilde{\Delta}_{0}\right|,
$$

which for $\beta=0$ holds, while for $\beta \neq 0$ will be satisfied when

$$
\ell \ll 1 \text {. }
$$

If $\omega=\pi / 2$, inequality (2.18) leads to

$$
\begin{aligned}
& \left|1+16 r_{5}+2 r_{1} \tilde{\Delta}_{0}+r_{6} \tilde{\Delta}_{0}^{2}-8\left(r_{3}+r_{9} \tilde{\Delta}_{0}\right)\right| \\
& \leq\left|1+4 r_{3}-r_{1} \tilde{\Delta}_{0}\right|,
\end{aligned}
$$

which subject to (2.20) holds.

\subsection{The Modified Predictor-Corrector Scheme}

To avoid solving the nonlinear system (2.14) the following Modified Predictor-Corrector (MPC) scheme is proposed.

\subsubsection{Predictor}

$\hat{\mathbf{U}}(t+\ell)$ is evaluated from the reccurence relation (2.12) replacing the matrix-exponential term with the following explicit second order rational approximant

$$
\hat{\mathbf{U}}(t+\ell)=\left(I+\ell D+\frac{1}{2} \ell^{2} D^{2}\right) \mathbf{U}(t)+\mathcal{O}\left(\ell^{2}\right) \text { as } \ell \rightarrow 0 .
$$

Then Equation (2.21) subject to Equation (2.11) using 
again the notations (2.7) - (2.8) leads to

$$
\begin{aligned}
& \hat{\mathbf{U}}(t+\ell) \\
= & \mathbf{U}(t)+\ell\left[-\alpha \Delta^{n} A+B+\beta \tilde{\Delta}^{n}\right] \mathbf{U}(t) \\
& +\frac{\ell^{2}}{2}\left[\left(\alpha \Delta^{n} A\right)^{2}+B^{2}+\left(\beta \tilde{\Delta}^{n}\right)^{2}-\alpha \Delta^{n} A B\right. \\
& -\alpha \beta \Delta^{n} A \tilde{\Delta}^{n}-\alpha B \Delta^{n} A+\beta B \tilde{\Delta}^{n} \\
& \left.-\alpha \beta \tilde{\Delta}^{n} \Delta^{n} A+\beta \tilde{\Delta}^{n} B\right] \mathbf{U}(t) .
\end{aligned}
$$

Let $p_{1}=\ell / h^{2}, p_{2}=\ell \alpha / 2 h, p_{3}=\ell^{2} \alpha^{2} / 8 h^{2}$,

$p_{4}=\ell^{2} \beta^{2} / 2, p_{5}=\ell^{2} / 2 h^{4}, p_{6}=\ell^{2} \alpha / 4 h^{3}$,

$p_{7}=\ell^{2} \alpha \beta / 4 h$ and $p_{8}=\ell^{2} \alpha / 4 h^{3}$.

Equation (2.22), when applied to the general mesh point of the grid $G$, gives

$$
\begin{aligned}
\hat{U}_{m}^{n+1}= & U_{m}^{n}+\ell \beta \tilde{\Delta}_{m}^{n} U_{m}^{n}+p_{1}\left(U_{m-1}^{n}-2 U_{m}^{n}+U_{m+1}^{n}\right) \\
& -p_{2} \Delta_{m}^{n}\left(U_{m+1}^{n}-U_{m-1}^{n}\right)+p_{3} \Delta_{m}^{n}\left[\Delta_{m-1}^{n} U_{m-2}^{n}\right. \\
& \left.-\left(\Delta_{m-1}^{n}+\Delta_{m+1}^{n}\right) U_{m}^{n}+\Delta_{m+1}^{n} U_{m+2}^{n}\right]+p_{4}\left(\tilde{\Delta}_{m}^{n}\right)^{2} U_{m}^{n} \\
& +p_{5}\left(U_{m-2}^{n}-4 U_{m-1}^{n}+6 U_{m}^{n}-4 U_{m+1}^{n}+U_{m+2}^{n}\right) \\
& -p_{6} \Delta_{m}^{n}\left(-U_{m-2}^{n}+2 U_{m-1}^{n}-2 U_{m+1}^{n}+U_{m+2}^{n}\right) \\
& -p_{7}\left(U_{m}^{n}\right)^{\delta}\left(\tilde{\Delta}_{m+1}^{n} U_{m+1}^{n}-\tilde{\Delta}_{m-1}^{n} U_{m-1}^{n}\right) \\
& -p_{6}\left[-\Delta_{m-1}^{n} U_{m-2}^{n}+2 \Delta_{m}^{n} U_{m-1}^{n}-2 \Delta_{m}^{n} U_{m+1}^{n}\right. \\
& \left.+\left(\Delta_{m-1}^{n}-\Delta_{m+1}^{n}\right) U_{m}^{n}+\Delta_{m+1}^{n} U_{m+2}^{n}\right] \\
& +p_{8}\left(\tilde{\Delta}_{m-1}^{n} U_{m-1}^{n}-2 \tilde{\Delta}_{m}^{n} U_{m}^{n}+\tilde{\Delta}_{m+1}^{n} U_{m+1}^{n}\right) \\
& -p_{7} \Delta_{m}^{n} \tilde{\Delta}_{m}^{n}\left(U_{m+1}^{n}-U_{m-1}^{n}\right) \\
& +p_{8} \tilde{\Delta}_{m}^{n}\left(U_{m-1}^{n}-2 U_{m}^{n}+U_{m+1}^{n}\right) ; \quad m=0,1, \ldots, N+1 .
\end{aligned}
$$

\section{Stability Analysis}

Following again the Fourier method of analysing stability Equation (2.23) leads to the following stability equation

$$
\begin{aligned}
\xi= & 1+\ell \beta \tilde{\Delta}_{0}+p_{4} \tilde{\Delta}_{0}^{2}-4\left(p_{1}+2 p_{8} \tilde{\Delta}_{0}\right) \sin ^{2} \omega \\
& +16 p_{5} \sin ^{4} \omega-4 p_{3} \Delta_{0}^{2} \sin ^{2} 2 \omega+2 i \Delta_{0} \\
& \times\left[\left(-p_{2}+4 p_{6}-2 p_{7} \tilde{\Delta}_{0}\right) \sin 2 \omega-4 p_{6} \sin 4 \omega\right],
\end{aligned}
$$

which is of the form (2.17) with $A=1$. Then condition (2.18) for $\omega=0$ leads to

$$
\left|1+\ell \beta \tilde{\Delta}_{0}+\frac{1}{2} \ell^{2} \beta^{2} \tilde{\Delta}_{0}^{2}\right| \leq 1 .
$$

which for $\beta=0$ is obvious, while for $\beta \neq 0$ is satis- fied when the condition (2.20) holds. When $\omega=\pi / 2$ condition (2.18) leads to

$$
\left|1+\left(-\frac{4}{h^{2}}+\beta \tilde{\Delta}_{0}\right) \ell+\left(\frac{8}{h^{4}}-\frac{4}{h^{2}} \beta \tilde{\Delta}_{0}+\frac{1}{2} \beta^{2} \tilde{\Delta}_{0}^{2}\right) \ell^{2}\right| \leq 1,
$$

which again subject to (2.20) is always satisfied.

\subsubsection{Corrector}

The corrector arises from Equation (2.13) as follows

$$
\begin{aligned}
\boldsymbol{U}(t+\ell) & =\left(\frac{1}{2} \ell D-\frac{1}{12} \ell^{2} D^{2}\right) \hat{\boldsymbol{U}}(t+\ell) \\
& +\left(I+\frac{1}{2} \ell D+\frac{1}{12} \ell^{2} D^{2}\right) \boldsymbol{U}(t) .
\end{aligned}
$$

Instead of the classical substitution of $\boldsymbol{U}(t+\ell)$ in the right-hand side of (2.27) by $\hat{\boldsymbol{U}}(t+\ell)$, a modified predictor-corrector method (MPC) was applied [5]. The MPC method, which is explicit and is applied once, consists of considering (2.27) component-wise and using an updated component in the corrector vector as soon as it becomes available. Hence, in computing $U_{m}^{n+1}$ the corrected value $U_{m-1}^{n+1}$ instead of the predicted value $\hat{U}_{m-1}^{n+1}$ is used. The stability analysis of the corrector is given in Section 2.2.1.

\section{Numerical Results}

For the linearization $U_{0}=\max _{m=0,1, \ldots, N+1}\left\{u_{m}^{0}\right\}$ was given. Let the error at time level $t=n \ell ; n=1,2, \cdots$ be $e=e(t)=L_{\infty}=\max _{m=0,1, \cdots, N+1}\left|u_{m}^{n}-U_{m}^{n}\right|$ and $x_{e}$ the $x$-coordinate at which $e$ occurs. Then $e_{(2.2)-(2.3)}$ denotes the error arising when using the boundary values (2.2) - (2.3), while analogous notations for the other boundary conditions are used. In all experiments the initial condition (1.2) was given by the value $f(x)=u(x, 0)$ with $u$ the theoretical solution (1.3). Experiments proved that the most accurate results are obtained for $h=0.1$ and $\ell=10^{-4}$. For reasons of comparison with the corresponding works in $[12,13,16,17]$ the same parameter values were used.

\subsection{Problem [12]}

From the experiments the following are deduced:

1) when $\alpha \neq 0$ (Table 1) using:

i) the boundary values $(2.2)-(2.3)$ the method introduced gives more accurate results for all time levels used than the corresponding results in [12] and marginally more accurate than those in $[13,17]$,

ii) the boundary condition (2.5) gives more accurate results than those in [12] and approximately equivalent to those in $[13,17]$. 
From (i) - (ii) it is deduced that the boundary values (2.2) - (2.3) give more accurate results than the boundary condition (2.5).

2) when $\alpha=0$ (Table 2) using the boundary values (2.2) - (2.3) the method introduced has given

-for $\delta=1$ more accurate results for all time levels used than the corresponding in [12], and

-for $\delta>1$ results with marginally inferior accuracy to those in [12].

In Figure 1(a) the solution $u$ for $t \in(0,1)$ and $x \in\left[-10^{4}, 10^{4}\right]$ is shown, while in Figure 1(b) the relevant solution $U$ when $x \in[0,1]$.

Table 1. Problem [12]. Comparisons of the proposed method for various values of $x, t$ with $\alpha=1, \beta=1, \gamma=0.001$ and $\delta=1(h$ $\left.=0.1, \ell=10^{-4}\right)$.

\begin{tabular}{cccccccc}
\hline$t$ & $x$ & Exact & $e_{(2.2)-(2.3)}$ & $e_{(2.5)}$ & $e[12]$ & $e[13]$ & $e[17]$ \\
\hline \multirow{2}{*}{0.05} & 0.1 & $0.5000187 \mathrm{E}-03$ & $1.87406 \mathrm{E}-08$ & $1.26463 \mathrm{E}-09$ & $1.93715 \mathrm{E}-07$ & $1.87406 \mathrm{E}-08$ & $1.87405 \mathrm{E}-08$ \\
& 0.5 & $0.5000687 \mathrm{E}-03$ & $1.87399 \mathrm{E}-08$ & $1.97698 \mathrm{E}-08$ & $1.93730 \mathrm{E}-07$ & $1.87406 \mathrm{E}-08$ & $1.87405 \mathrm{E}-08$ \\
& 0.9 & $0.5001187 \mathrm{E}-03$ & $1.87250 \mathrm{E}-08$ & $4.60177 \mathrm{E}-08$ & $1.93745 \mathrm{E}-07$ & $1.87406 \mathrm{E}-08$ & $1.87405 \mathrm{E}-08$ \\
& 0.1 & $0.5000250 \mathrm{E}-03$ & $3.74813 \mathrm{E}-08$ & $6.39532 \mathrm{E}-09$ & $3.87434 \mathrm{E}-07$ & $3.74812 \mathrm{E}-08$ & $3.74813 \mathrm{E}-08$ \\
0.1 & 0.5 & $0.5000750 \mathrm{E}-03$ & $3.74736 \mathrm{E}-08$ & $3.99558 \mathrm{E}-08$ & $3.87464 \mathrm{E}-07$ & $3.74812 \mathrm{E}-08$ & $1.37481 \mathrm{E}-08$ \\
& 0.9 & $0.5001250 \mathrm{E}-03$ & $3.74186 \mathrm{E}-08$ & $7.66328 \mathrm{E}-08$ & $3.87494 \mathrm{E}-07$ & $3.74812 \mathrm{E}-08$ & $3.74813 \mathrm{E}-08$ \\
& 0.1 & $0.5001374 \mathrm{E}-03$ & $3.74814 \mathrm{E}-07$ & $3.29223 \mathrm{E}-07$ & $3.87501 \mathrm{E}-06$ & $3.74812 \mathrm{E}-07$ & $3.74812 \mathrm{E}-07$ \\
\multirow{2}{*}{1} & 0.5 & $0.5001874 \mathrm{E}-03$ & $3.72103 \mathrm{E}-07$ & $3.79222 \mathrm{E}-07$ & $3.87531 \mathrm{E}-06$ & $3.74812 \mathrm{E}-07$ & $3.74813 \mathrm{E}-07$ \\
& 0.9 & $0.5002374 \mathrm{E}-03$ & $3.68427 \mathrm{E}-07$ & $4.29222 \mathrm{E}-07$ & $3.87561 \mathrm{E}-06$ & $3.74812 \mathrm{E}-07$ & $3.74813 \mathrm{E}-07$ \\
\hline
\end{tabular}

Table 2. Problem [12]. Comparisons of the proposed method for various values of $x, t$ and $\delta$ with $\alpha=0, \beta=1$ and $\gamma=0.001(h$ $=0.1, \ell=10^{-4}$ ).

\begin{tabular}{cccccccc}
\hline \multirow{2}{*}{$t$} & $x$ & \multicolumn{3}{c}{$\delta=1$} & \multicolumn{3}{c}{$\delta=2$} \\
\cline { 3 - 8 } & & $e_{(2.2)-(2.3)}$ & $e[12]$ & $e_{(2.2)-(2.3)}$ & $e[12]$ & $e_{(2.2)-(2.3)}$ & $e[12]$ \\
\hline \multirow{3}{*}{0.05} & 0.1 & $2.49875 \mathrm{E}-08$ & $1.87465 \mathrm{E}-07$ & $1.11763 \mathrm{E}-06$ & $5.58901 \mathrm{E}-07$ & $3.96731 \mathrm{E}-06$ & $1.9841 \mathrm{E}-06$ \\
& 0.5 & $2.49875 \mathrm{E}-08$ & $1.87486 \mathrm{E}-07$ & $1.11750 \mathrm{E}-06$ & $5.58836 \mathrm{E}-07$ & $3.96652 \mathrm{E}-06$ & $1.98371 \mathrm{E}-06$ \\
& 0.9 & $2.49874 \mathrm{E}-08$ & $1.87508 \mathrm{E}-07$ & $1.11737 \mathrm{E}-06$ & $5.58772 \mathrm{E}-07$ & $3.96572 \mathrm{E}-06$ & $1.98331 \mathrm{E}-06$ \\
& 0.1 & $4.99750 \mathrm{E}-08$ & $3.74934 \mathrm{E}-07$ & $2.23526 \mathrm{E}-06$ & $1.11779 \mathrm{E}-06$ & $7.93462 \mathrm{E}-06$ & $3.96811 \mathrm{E}-06$ \\
0.1 & 0.5 & $4.99750 \mathrm{E}-08$ & $3.74977 \mathrm{E}-07$ & $2.23500 \mathrm{E}-06$ & $1.11766 \mathrm{E}-06$ & $7.93304 \mathrm{E}-06$ & $3.96731 \mathrm{E}-06$ \\
& 0.9 & $4.99749 \mathrm{E}-08$ & $3.75019 \mathrm{E}-07$ & $2.23474 \mathrm{E}-06$ & $1.11753 \mathrm{E}-06$ & $7.93144 \mathrm{E}-06$ & $3.96652 \mathrm{E}-06$ \\
& 0.1 & $4.99750 \mathrm{E}-07$ & $3.75002 \mathrm{E}-06$ & $2.23526 \mathrm{E}-05$ & $1.11754 \mathrm{E}-05$ & $7.93462 \mathrm{E}-05$ & $3.96632 \mathrm{E}-05$ \\
& 0.5 & $4.99749 \mathrm{E}-07$ & $3.75044 \mathrm{E}-06$ & $2.23500 \mathrm{E}-05$ & $1.00741 \mathrm{E}-05$ & $7.93303 \mathrm{E}-05$ & $3.96553 \mathrm{E}-05$ \\
& 0.9 & $4.99749 \mathrm{E}-07$ & $3.75086 \mathrm{E}-06$ & $2.23474 \mathrm{E}-05$ & $1.11728 \mathrm{E}-05$ & $7.93143 \mathrm{E}-05$ & $3.96473 \mathrm{E}-05$ \\
\hline
\end{tabular}

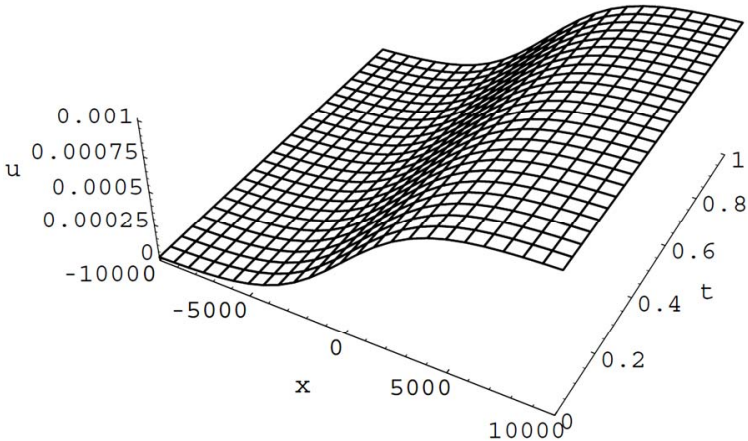

(a)

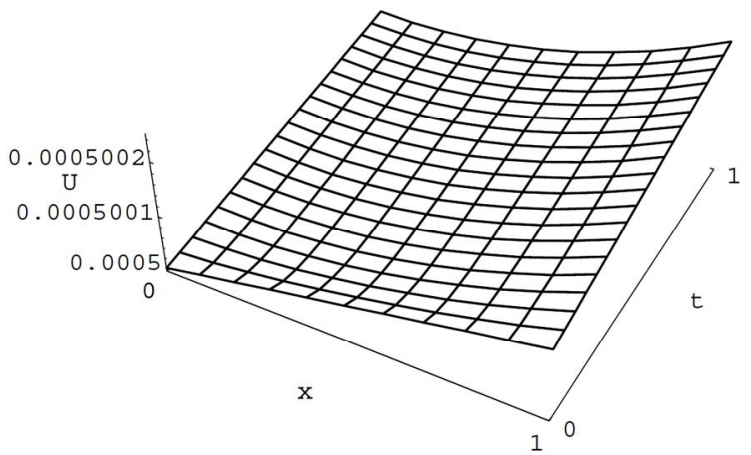

(b)

Figure 1. Problem [12] with $\delta=1, \alpha=1, \beta=1, \gamma=0.001$ when $t \in[0,1]$ : In (a) the surface shows $u(x, t)$ for $x \in\left[-10^{4}, 10^{4}\right]$, while in (b) the numerical solution $U$ when $x \in[0,1]$. 
Table 3. Problem [17]. Comparisons of the proposed method for various values of $\delta$ and $\gamma$ when $\alpha=\beta=1\left(h=0.1, \ell=10^{-4}\right)$.

\begin{tabular}{|c|c|c|c|c|c|c|c|c|}
\hline$t=1$ & $\delta=1$ & $\gamma=10^{-3}$ & $t=0.5$ & $\delta=2$ & $\gamma=10^{-2}$ & $t=0.5$ & $\delta=4$ & $\gamma=10^{-2}$ \\
\hline$x$ & $e_{(2.2)-(2.3)}$ & $e[17]$ & $x$ & $e_{(2.2)-(2.3)}$ & $e[17]$ & $x$ & $e_{(2.2)-(2.3)}$ & $e[17]$ \\
\hline 0.1 & $3.74814 \mathrm{E}-07$ & $3.74812 \mathrm{E}-07$ & 0.1 & $3.89463 \mathrm{E}-05$ & $2.75734 \mathrm{E}-04$ & 0.1 & $5.69322 \mathrm{E}-05$ & $1.08762 \mathrm{E}-03$ \\
\hline 0.5 & $3.72103 \mathrm{E}-07$ & $3.74814 \mathrm{E}-07$ & 0.3 & $3.89656 \mathrm{E}-05$ & $2.75614 \mathrm{E}-04$ & 0.3 & $5.69778 \mathrm{E}-05$ & $1.08644 \mathrm{E}-03$ \\
\hline 0.9 & $3.68427 \mathrm{E}-07$ & $3.74813 \mathrm{E}-07$ & 0.5 & $3.89844 \mathrm{E}-05$ & $2.75493 \mathrm{E}-04$ & 0.5 & $5.70134 \mathrm{E}-05$ & $1.08527 \mathrm{E}-03$ \\
\hline
\end{tabular}

Table 4. Problem [16]. Boundary conditions (2.4) - (2.5). Comparisons of the proposed method for various values of $t$ with $\alpha=$ 5 and $\delta=1\left(h=0.1, \ell=10^{-4}\right)$.

\begin{tabular}{cccccccc}
\hline \multirow{2}{*}{$t$} & $\beta$ & \multicolumn{3}{c}{$\gamma=10^{-3}$} & \multicolumn{2}{c}{$\gamma=10^{-4}$} & \multicolumn{2}{c}{$\gamma=10^{-5}$} \\
\cline { 2 - 7 } & & Method & $e[16]$ & Method & $e[16]$ & $e$ & $e[16]$ \\
\hline \multirow{2}{*}{0.3} & 1 & $3.1570 \mathrm{E}-08$ & $3.1616 \mathrm{E}-08$ & $3.1584 \mathrm{E}-10$ & $3.1630 \mathrm{E}-10$ & $3.3410 \mathrm{E}-12$ & $3.1632 \mathrm{E}-12$ \\
& 10 & $3.9684 \mathrm{E}-07$ & $3.9742 \mathrm{E}-07$ & $3.9702 \mathrm{E}-09$ & $3.9760 \mathrm{E}-09$ & $3.9704 \mathrm{E}-11$ & $3.9762 \mathrm{E}-11$ \\
& 100 & $5.0291 \mathrm{E}-06$ & $5.0365 \mathrm{E}-06$ & $5.0316 \mathrm{E}-08$ & $5.0389 \mathrm{E}-08$ & $5.0318 \mathrm{E}-10$ & $5.0392 \mathrm{E}-10$ \\
& 1 & $3.3393 \mathrm{E}-08$ & $3.3394 \mathrm{E}-08$ & $3.3408 \mathrm{E}-10$ & $3.3409 \mathrm{E}-10$ & $3.3410 \mathrm{E}-12$ & $3.3411 \mathrm{E}-12$ \\
& 10 & $4.1976 \mathrm{E}-07$ & $4.1977 \mathrm{E}-07$ & $4.1995 \mathrm{E}-09$ & $4.1996 \mathrm{E}-09$ & $4.1997 \mathrm{E}-11$ & $4.1998 \mathrm{E}-11$ \\
& 100 & $5.3165 \mathrm{E}-06$ & $5.3166 \mathrm{E}-06$ & $5.3221 \mathrm{E}-08$ & $5.3223 \mathrm{E}-08$ & $5.3224 \mathrm{E}-10$ & $5.3225 \mathrm{E}-10$ \\
\hline
\end{tabular}

\subsection{Problem [17]}

From Table 3 it is deduced that the method introduced using the boundary values (2.2) - (2.3) has given more accurate results for all time levels and parameters used than the relevant method in [17].

\subsection{Problem [16]}

For reasons of comparison with the relevant results in [18] the boundary conditions $(2.4)-(2.5)$ with $g_{0}(t)=$ $u(0, t)$ and $g_{1}(t)=u(1, t)$ were used. From Table 4 it is deduced that the proposed method:

-has given marginally more accurate results to those in [16] for all time levels and $\beta, \gamma$ used,

-for fixed $\alpha, \delta$ and

- $\beta$, the accuracy increases and $U$ tends to identify with $u$ at long time level as $\gamma$ is refined,

- $\gamma$, as $\beta$ increases, the accuracy decreases.

\section{Conclusions}

An implicit finite difference scheme based on fourthorder rational approximants to the matrix exponential term was proposed for the numerical solution of the Burgers-Huxley equation. The resulting nonlinear scheme was solved using an improved predictor-corrector method. The computational efficiency of the proposed method given in detail in Section 3 was tested by comparing the numerical results to selected ones in $[12,13,16,17]$ using both appropriate boundary values and conditions. Conclusions for the boundaries used were derived.
Since the real world problems lead to the numerical solution of nonlinear equations or systems of equations, the introduced low cost and easy-to-handle method enables us to obtain accurate solutions.

\section{References}

[1] A. L. Hodgkin and A. F. Huxley, "A Quantitative Description of Ion Currents and Its Applications to Conduction and Excitation in Nerve Membranes," Journal of Physiology, Vol. 117, No. 4, 1952, pp. 500-544.

[2] J. Satsuma, "Topics in Soliton Theory and Exactly Solvable Nonlinear Equations," In: M. Ablowitz, B. Fuchssteiner and M. Kruskal, Eds., World Scientific, Singapore City, 1987.

[3] X. Wang, "Nerve Propagation and Wall in Liquid Crystals," Physics Letters, Vol. 112A, No. 8, 1985, pp. 402-406.

[4] X. Y. Wang, Z. S. Zhu and Y. K. Lu, "Solitary Wave Solutions of the Generalised Burgers-Huxley Equation," Journal of Physics A: Mathematical and General, Vol. 23, No. 3, 1990, pp. 271-274.

doi:10.1088/0305-4470/23/3/011

[5] A. G. Bratsos, "A Fourth-Order Numerical Scheme for Solving the Modified Burgers Equation," Computers and Mathematics with Applications, Vol. 60, No. 5, 2010, pp. 1393-1400. doi:10.1016/j.camwa.2010.06.021

[6] R. Fitzhugh, "Mathematical Models of Excitation and Propagation in Nerve," In: H. P. Schwan. Ed., Biological Engineering, McGraw Hill, New York, 1969, pp. 1-85.

[7] P. G. Estévez and P. R. Gordoa, "Painlevé Analysis of the generalized Burgers-Huxley Equation," Journal of Physics A: Mathematical and General, Vol. 23, No. 21, 1990, 
pp. 4831-4837.

[8] P. G. Estévez, "Non-Classical Symmetries and the Singular Manifold Method: The Burgers and the Burgers-Huxley Equations," Journal of Physics A: Mathematical and General, Vol. 27, No. 6, 1994, pp. 21132127.

[9] O. Yu. Yefimova and N. A. Kudryashov, "Exact Solutions of the Burgers-Huxley Equation," Journal of Applied Mathematics and Mechanics, Vol. 68, No. 3, 2004, pp. 413-420. doi:10.1016/S0021-8928(04)00055-3

[10] X. Deng, "Travelling Wave Solutions for the Generalized Burgers-Huxley Equation," Applied Mathematics and Computation, Vol. 204, No. 1, 2008, pp. 733-737. doi:10.1016/j.amc.2008.07.020

[11] A. M. Wazwaz, "Analytic Study on Burgers, Fisher, Huxley Equations and Combined Forms of These Equations," Applied Mathematics and Computation, Vol. 195, No. 2, 2008, pp. 754-761. doi:10.1016/j.amc.2007.05.020

[12] H. N. A. Ismail, K. Raslan and A. A. A. Rabboh, "Adomian Decomposition Method for Burger's Huxley and Burger's-Fisher Equations," Applied Mathematics and Computation, Vol. 159, No. 1, 2004, pp. 291-301. doi:10.1016/j.amc.2003.10.050

[13] I. Hashim, M. S. M. Noorani and M. R. S. Al-Hadidi, "Solving the Generalized Burgers-Huxley Equation Using the Adomian Decomposition Method," Mathematical and Computer Modelling, Vol. 43, No. 11-12, 2006, pp. 1404-1411. doi:10.1016/j.mcm.2005.08.017

[14] M. Javidi, "A Numerical Solution of the Generalized Burger's-Huxley Equation by Pseudospectral Method and
Darvishi's Preconditioning," Applied Mathematics and Computation, Vol. 175, No. 1, 2006, pp. 1619-1628. doi:10.1016/j.amc.2005.09.009

[15] M. Javidi, "A Numerical Solution of the Generalized Burgers-Huxley Equation by Spectral Collocation Method," Applied Mathematics and Computation, Vol. 178, No. 2, 2006, pp. 338-344.

[16] M. Javidi and A. Golbabai, "A New Domain Decomposition Algorithm for Generalized Burgers-Huxley Equation Based on Chebyshev Polynomials and Preconditioning," Chaos Soliton \& Fractals, Vol. 39, 2009, pp. 849-857.

[17] B. Batiha, M. S. M. Noorani and I. Hashim, "Application of Variational Iteration Method to the Generalized Burgers-Huxley Equation," Chaos Solitons \& Fractals, Vol. 36, No. 3, 2008, pp. 660-663. doi:10.1016/j.chaos.2006.06.080

[18] A. Khattak, "A Computational Meshless Method for the Generalized Burgers-Huxley Equation," Applied Mathematical Modelling, Vol. 33, No. 9, 2009, pp. 3718-3729. doi:10.1016/j.apm.2008.12.010

[19] E. Babolian and J. Saeidian, "Analytic Approximate Solutions to Burgers, Fisher, Huxley Equations and Two Combined Forms of These Equations," Communications in Nonlinear Science and Numerical Simulation, Vol. 14, 2009, pp. 1984-1992. doi:10.1016/j.cnsns.2008.07.019

[20] B. Fornberg, "A Practical Guide to Pseudospectral Methods," Cambridge University Press, Cambridge, 1998.

[21] E. H. Twizell, "Computational Methods for Partial Differential Equations," Ellis Horwood Limited, Chichester, 1984. 